\title{
Cox Regression Analysis of Dependent Interval-Censored Failure Time Data
}

\author{
Ling $\mathrm{Ma}^{\mathrm{a}, *}$, Tao Hu${ }^{\mathrm{b}}$, Jianguo Sun ${ }^{\mathrm{a}}$ \\ ${ }^{a}$ Department of Statistics, University of Missouri, Columbia, MO, United States \\ ${ }^{b}$ School of Mathematical Sciences and BCMIIS, Capital Normal University, Beijing, China
}

\begin{abstract}
Many procedures have been proposed for regression analysis of interval-censored failure time data arising from the Cox or proportional hazards model. However, most of these existing methods only apply to the situation where the censoring mechanism generating censoring intervals is independent of the failure time of interest, and it is well-known that sometimes this may not be true in practice. To address this issue, a new approach that allows the dependence between the censoring mechanism and the failure time is proposed. More specifically, a situation where the dependence is through the length of censoring intervals is considered as it is often the case in follow-up studies. The asymptotic properties of the proposed estimators are established and the numerical studies are conducted for the assessment of the finite sample properties of the estimators.

Keywords: Copula model; Cox model; Dependent interval-censoring; Efficient semiparametric estimation
\end{abstract}

\section{Introduction}

This paper discusses regression analysis of interval-censored failure time data arising from the Cox or proportional hazards model. By interval-censored data, we mean that instead of being observed exactly, the failure time of interest is observed only to belong to an interval, commonly denoted by $(L, R]$ or $L<T \leq R$ (Chen et aL, 2012; Finkelstein, 1986; Huang, 1996; Sinn, 20106). That is, the occurrence of the failure event of interest is known only to be within the interval $(L, R]$. Examples of the areas that often produce such data include health or medical follow-up studies such

\footnotetext{
* Corresponding author

Email address: mlbegood@gmail.com (Ling Ma)
} 
as clinical trials as well as social sciences. In addition, it is easy to see that the interval-censored data include right-censored data, whose analysis has been extensively discussed in the literature, as a special case (KalbHeisch and Prentice, 20102).

The analysis of interval-censored failure time data has been attracting more and more attention recently and especially, many methods have been developed for their regression analysis under various regression models including the proportional hazards model. For example, a pioneering work was given by Finkelstein (ㅍ6), which discussed the fitting of the Cox model to the data, and for the same problem, Goggins et al. (11998) and Zhang et al. (2010) developed a Markov chain Monte Carlo EM algorithm and a spline-based maximum likelihood approach, respectively. Also among others, Zhang et al. (2010.5) proposed an estimating equation procedure for fitting the linear transformation model to interval-censored data. More and relatively complete references on this can be found in Chen et al. (2012) and Sun (2006).

For the analysis of interval-censored failure time data, it is easy to see that in addition to the failure time $T$ of interest, one may need to concern the mechanism that generates $L$ and $R$ also. For this, most of the existing methods assume the noninformative or independent mechanism under which one can carry out the conditional analysis given $L<T \leq R$. On the other hand, it is apparent that this may not be true in some situations. In a clinical trial with the follow-up of both healthy subjects and patients, for example, the patients may tend to pay more clinical visits than the scheduled ones. Several methods have been proposed in the literature for the analysis of dependent interval-censored data. For example, Finkelstein et al. (2002) and Betensky and Finkelstein (20022) considered the one-sample problem related to such data, and Zhang et al. (2007) gave a latent variable-based estimation procedure for regression analysis of dependent interval-censored data from the Cox model. Note that the method in Zhang et al. (2007) used a log-normal frailty to describe the dependence structure and it is apparent that in reality, it may be difficult to check such specific dependence structure. In other words, a more general model or procedure would be clearly needed.

In the following, we will discuss the same problem considered as Zhang et al. (2007) and present 35 a more general, copula model-based approach. More specifically, we will assume that the pair $(L, R]$ is generated from an underline observation process and focus on the case where the dependence between the failure time and the censoring mechanism can be described by the length of censoring intervals. One situation where this can happen is medical follow-up studies where subjects may 
pay more or less clinical visits. For the analysis, a maximum likelihood estimation procedure will be developed with the use of $I$-spline functions (Ramsay, 1988; Lu et al, 2007). Both the model and the method will be described in the next section. In Section 3, some asymptotic properties of the proposed estimators are established, including the consistency and asymptotic normality of the estimated regression parameters, and the variance estimation is discussed. Some simulation results are presented in Section 4 and Section 5 provides an illustrative example. Finally Section 6 contains some discussion and concluding remarks.

\section{Semiparametric Maximum Likelihood Estimation}

Consider a failure time study that involves $n$ independent subjects and yields only intervalcensored failure time data. For subject $i$, let $T_{i}$ denote the failure time of interest and $Z_{i}$ the vector of covariates, and suppose that there exists an array of underlying observation times $\boldsymbol{E}_{i}=\left\{E_{i, j}\right.$ : $j=0,1,2, \ldots\}$ such that $E_{i, j}<E_{i, j^{\prime}}$ for any $j<j^{\prime}$. Define $W_{i, j}=E_{i, j}-E_{i, j-1}, j=1,2, \ldots$, the gap times of the observation process with $E_{i 0}=0$ and assume that given $Z_{i}$, the $W_{i, j}$ 's follow the same distribution. Furthermore assume that $T_{i}$ may depend on the underlying observation process through the gap times and that given $Z_{i},\left\{\left(T_{i}, W_{i, j}\right), j=1,2, \ldots\right\}$ follow the same joint distribution. In the following, for each $i$, define $\left(\tilde{L}_{i}, \tilde{R}_{i}\right]$ to be the random interval among $\left(0, E_{i, 1}\right],\left(E_{i, 1}, E_{i, 2}\right], \ldots$ that contains $T_{i}$. Note that the mechanism behind the observation process and censoring intervals here is similar to that behind the mixed case $k$ interval-censored data (Shick and Yu, 2000); Sun, 20106).

In practice such as in medical follow-up studies, there usually exists an administrative censoring time $\zeta_{i}$ beyond which the observation process is no longer available. For each $i$, define $L_{i}=$ $\max \left\{E_{i, j}: E_{i, j}<\min \left(\zeta_{i}, T_{i}\right), j=0,1,2, \ldots\right\}$ and $R_{i}=\min \left\{E_{i, j}: E_{i, j}>L_{i}, j=1,2, \ldots\right\}$. Also, define $\delta_{i}=1$ if $R_{i} \leq \zeta_{i}$ and $\delta_{i}=0$ otherwise. Note that when $\delta_{i}=0, R_{i}$ is not observed but right-censored at $\zeta_{i}$ and $T_{i}$ is right-censored. For ease of explanation, we define $\left(\tilde{L}_{i}, \tilde{R}_{i}\right]$ as covering interval and $\left(L_{i}, R_{i}\right]$ as 'observed interval' (even though $R_{i}$ is not directly observed for right-censored subjects). And define $W_{i}=R_{i}-L_{i}$ as the 'observed interval length'. It is easy to see that if $\delta_{i}=1$, $W_{i}$ is exactly observed and $T_{i}$ lies in the observed interval. For subjects with $\delta_{i}=0$, note that $L_{i}$ is not necessarily equal to $\tilde{L}_{i}$ and also $\left(L_{i}, R_{i}\right]$ may not contain $T_{i}$. In fact, since $R_{i}$ is right-censored at $\zeta_{i}$, we only observe that $T_{i}>L_{i}$ and $W_{i}>\zeta_{i}-L_{i}$. An illustrative example for this situation with $\delta_{i}=0$ is given in Figure 1. Assume that given $Z_{i}$ and $T_{i}$ belonging to the censoring interval, 
the distribution of $T_{i}$ depends on the censoring interval only through its length. That is, we have $\operatorname{Pr}\left(T_{i} \leq t \mid L_{i}<T_{i} \leq R_{i} ; L_{i}=l_{i}, R_{i}=r_{i}, Z_{i}=z_{i}\right)=\operatorname{Pr}\left(T_{i} \leq t \mid l_{i}<T_{i} \leq r_{i} ; W_{i} \equiv r_{i}-l_{i}, Z_{i}=z_{i}\right)$.

Then the likelihood function has the form

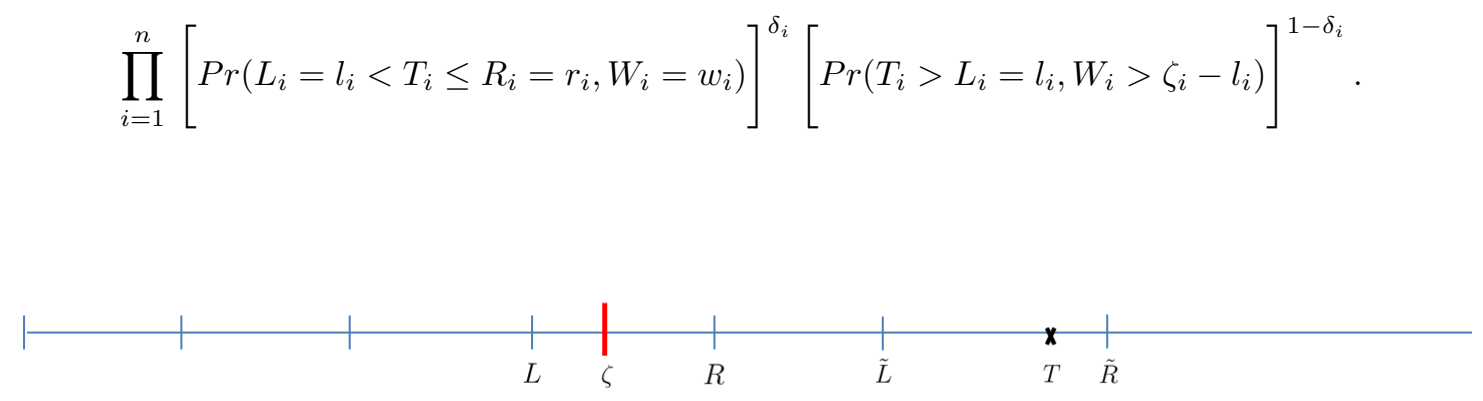

Figure 1: An illustrative example of right-censored observations.

Let $K$ denote the joint distribution of $T$ and $W$ given $Z$. Then it is well-known (Nelsen, [2006) that there exists a copula function $C_{\alpha}(u, v)$ defined on $I^{2}=[0,1] \times[0,1]$ such that

$$
K(t, w)=C_{\alpha}\left(F_{T}(t), F_{W}(w)\right), t \geq 0, w \geq 0
$$

In the above, $F_{T}$ and $F_{W}$ denote the marginal distributions of the $T$ and $W$ given $Z$, respectively, $\alpha$, usually referred to as the association parameter, represents the relationship between $T$ and $W$, and $C_{\alpha}(u, 0)=C_{\alpha}(0, v)=0, C_{\alpha}(u, 1)=u$ and $C_{\alpha}(1, v)=v$. Define $m_{\alpha}\left(F_{T}(t), F_{W}(w)\right)=P(T \leq$ $t \mid W=w, Z)$. Then we have

$$
m_{\alpha}\left(F_{T}(t), F_{W}(w)\right)=\left.\frac{\partial C_{\alpha}(u, v)}{\partial v}\right|_{u=F_{T}(t), v=F_{W}(w)}
$$

For the covariate effects, we will consider the following marginal Cox hazard models

$$
\lambda_{T}\left(t \mid Z_{i}\right)=\lambda_{1}(t) \exp \left(Z_{i}^{\prime} \beta\right), \lambda_{W}\left(w \mid Z_{i}\right)=\lambda_{2}(w) \exp \left(Z_{i}^{\prime} \gamma\right)
$$

for $T_{i}$ and $W_{i}$ given $Z_{i}$, respectively. Here $\lambda_{1}(t)$ and $\lambda_{2}(w)$ are unknown baseline hazard functions and $\beta$ and $\gamma$ denote vectors of regression parameters.

It is worth noting that the copula model formulation described above has been commonly used in multivariate failure time data analysis (Hougaard, 20001$)$ as well as the analysis of univariate failure time data in the presence of informative censoring. For example, by using the formulation, Zheng 
and Klein (11995) discussed the estimation of a marginal survival function based on right-censored data in the presence of dependent competing risks, and Chen (2010) considered the regression analysis of the same data. In particular, they showed that without prior or extra information, the association parameter $\alpha$ is not identifiable given the copula function. In the following, following these authors, we will assume that both the copula function and $\alpha$ are known and our main goal is to estimate regression parameters $\beta$ and $\gamma$.

For estimation of $\beta$ and $\gamma$, first note that it follows from the models defined above that we have $F_{T_{i}}(t)=F_{T}\left(t \mid Z_{i}\right)=1-\exp \left(-\Lambda_{1}(t) \exp \left(Z_{i}^{\prime} \beta\right)\right)$ and $F_{W_{i}}(w)=F_{W}\left(w \mid Z_{i}\right)=1-\exp \left(-\Lambda_{2}(w) \exp \left(Z_{i}^{\prime} \gamma\right)\right)$. Define $\theta=\left(\beta, \gamma, \Lambda_{1}(t), \Lambda_{2}(w)\right)$. Hence the likelihood function $L(\theta)$ can be rewritten as

$$
\begin{aligned}
L(\theta) & =\prod_{i=1}^{n}\left\{\left(\left[m_{\alpha}\left\{F_{T_{i}}\left(r_{i}\right), F_{W_{i}}\left(w_{i}\right)\right\}-m_{\alpha}\left\{F_{T_{i}}\left(l_{i}\right), F_{W_{i}}\left(w_{i}\right)\right\}\right] f_{W_{i}}\left(w_{i}\right)\right)^{\delta_{i}}\right. \\
& \left.\times\left(1-F_{W_{i}}\left(\zeta_{i}-l_{i}\right)-F_{T_{i}}\left(l_{i}\right)+C_{\alpha}\left\{F_{T_{i}}\left(l_{i}\right), F_{W_{i}}\left(\zeta_{i}-l_{i}\right)\right\}\right)^{1-\delta_{i}}\right\},
\end{aligned}
$$

where $f_{W_{i}}\left(w_{i}\right)=\lambda_{2}\left(w_{i}\right) \exp \left(Z_{i}^{\prime} \gamma\right) \exp \left\{-\Lambda_{2}\left(w_{i}\right) \exp \left(Z_{i}^{\prime} \gamma\right)\right\}$.

To estimate the regression parameters or $\theta$, it is apparent that a natural approach is to directly maximize the $\log$ likelihood function $l(\theta)=\log L(\theta)$. However, it is easy to see that this would not be straightforward as one has to deal with unknown functions $\Lambda_{1}$ and $\Lambda_{2}$. Instead we propose to approximate the two functions by $I$-spline functions first and then maximize the resulting sieve maximum likelihood function. Similar approaches have been used by Zhang et al. (2010) and others. More specifically, let $\left\{I_{j}(t)\right\}_{j=1}^{m+k_{n}}$ denote the $I$-spline base functions with order $m$ and $k_{n}$ interior knots, where $k_{n}=o\left(n^{\nu}\right)$ with $0<\nu<0.5$. Define $\varphi=\left(\Lambda_{1}, \Lambda_{2}\right)$ and

$$
\Theta_{n}=\left\{\theta_{n}=\left(\beta, \gamma, \varphi_{n}\right): \varphi_{n}=\left(\Lambda_{1 n}, \Lambda_{2 n}\right)\right\}=\mathcal{B} \otimes \mathcal{M}_{n}^{1} \otimes \mathcal{M}_{n}^{2}
$$

In the above, $\mathcal{B}=\left\{\left(\beta, \gamma \in R^{2 p},\|\beta\|+\|\gamma\| \leq M\right\}\right.$,

$$
\begin{gathered}
\mathcal{M}_{n}^{1}=\left\{\Lambda_{1 n}: \Lambda_{1 n}(t)=\sum_{j=1}^{m+k_{n}} \xi_{j} I_{j 1}(t), \xi_{j} \geq 0, j=1, \ldots, m+k_{n}, t \in\left[l_{t}, u_{t}\right]\right\}, \\
\mathcal{M}_{n}^{2}=\left\{\Lambda_{2 n}: \Lambda_{2 n}(w)=\sum_{j=1}^{m+k_{n}} \eta_{j} I_{j 2}(w), \eta_{j} \geq 0, j=1, \ldots, m+k_{n}, w \in\left[l_{w}, u_{w}\right]\right\},
\end{gathered}
$$

where $M$ is a positive constant, $\left[l_{t}, u_{t}\right]$ and $\left[l_{w}, u_{w}\right]$ denote the ranges of $\left\{l_{i}, r_{i}, i=1,2, \ldots, n\right\}$ and $\left\{\min \left(w_{i}, \zeta_{i}-l_{i}\right), i=1,2, \ldots, n\right\}$, respectively. In other words, instead of the original parameter space, we can focus on the space $\Theta_{n}$ when maximizing the log likelihood function $l(\theta)$. 
To see the validity of the space $\Theta_{n}$, for a vector $a$ and a function $f$, let $\|a\|$ denote the Euclidean norm and $\|f\|_{\infty}=\sup _{t}|f(t)|$, the supremum norm. Also for a random variable $X$ with the probability measure $P$, define $\|f(X)\|_{2}=\left(\int f^{2} d P\right)^{1 / 2}$, the $L^{2}(P)$ norm, and

$$
d^{2}\left(\theta^{1}, \theta^{2}\right)=\left\|\beta^{1}-\beta^{2}\right\|^{2}+\left\|\gamma^{1}-\gamma^{2}\right\|^{2}+\left\|\Lambda_{1}^{1}-\Lambda_{1}^{2}\right\|_{2}^{2}+\left\|\Lambda_{2}^{1}-\Lambda_{2}^{2}\right\|_{2}^{2},
$$

for $\theta^{i}=\left(\beta^{i}, \gamma^{i}, \Lambda_{1}^{i}, \Lambda_{2}^{i}\right), i=1,2$. Then it is easy to see that for any $\theta \in \Theta$, there exists a sequence of $\theta_{n}=\left(\beta, \gamma, \Lambda_{1 n}, \Lambda_{2 n}\right) \in \Theta_{n}$ such that $d\left(\theta, \theta_{n}\right) \leq c\left(\left\|\Lambda_{1}-\Lambda_{1 n}\right\|_{\infty}+\left\|\Lambda_{2}-\Lambda_{2 n}\right\|_{\infty}\right)=O\left(n^{-r \nu}\right)$ for some constant $c$ and $r=m+\eta$, where $\eta$ is defined in the condition (A4) given in the Appendix. It thus follows from Lemma A1 of Lil et al (20107) that $\Theta_{n}$ can be used as a sieve space of $\Theta$, and for estimation of $\theta$, we can define the estimator $\hat{\theta}=\left(\hat{\beta}, \hat{\gamma}, \hat{\Lambda}_{1 n}(\cdot), \hat{\Lambda}_{2 n}(\cdot)\right)$ as the value of $\theta$ that maximizes the log likelihood function $l(\theta)$ over $\Theta_{n}$. We will establish the asymptotic properties of $\hat{\theta}$ in the next section.

\section{Asymptotic Properties}

In this section, we establish the asymptotic properties of $\hat{\theta}$. First we give the consistency and the convergence rate of $\hat{\theta}$ and then the asymptotic normality of $\hat{\beta}$ and $\hat{\gamma}$. All limits below are taken as $n \rightarrow \infty$. Let $\theta_{0}=\left(\beta_{0}, \gamma_{0}, \Lambda_{10}, \Lambda_{20}\right)$ denote the true value of $\theta$.

Theorem 1. Suppose that the conditions A1-A4 described in the Appendix hold. Then $\hat{\beta}$ and $\hat{\gamma}$ are strong consistent estimators of $\beta_{0}$ and $\gamma_{0}$ and furthermore, we have $\left\|\hat{\Lambda}_{1 n}-\Lambda_{10}\right\|_{2} \longrightarrow 0$ and $\left\|\hat{\Lambda}_{2 n}-\Lambda_{20}\right\|_{2} \longrightarrow 0$ almost surely.

Theorem 2. Suppose that the conditions A1-A4 described in the Appendix hold. Then we have $\left\|\hat{\Lambda}_{1 n}-\Lambda_{10}\right\|_{2}+\left\|\hat{\Lambda}_{2 n}-\Lambda_{20}\right\|_{2}=O_{p}\left(n^{-(1-\nu) / 2}+n^{-r \nu}\right)$.

Theorem 3. Assume that $r>2$ and the conditions A1-A5 described in the Appendix hold.

90 Then we have $n^{1 / 2}\left(\left(\hat{\beta}-\beta_{0}\right)^{\prime},\left(\hat{\gamma}-\gamma_{0}\right)^{\prime}\right)^{\prime} \rightarrow N(0, \Sigma)$ in distribution and furthermore, $\left(\hat{\beta}^{\prime}, \hat{\gamma}^{\prime}\right)^{\prime}$ is semiparametrically efficient, where $\Sigma$ is given in the Appendix.

Note that by approximating the original parameter space by the $I$-spline function space, we change the original maximization problem to a much easy one over a finite parameter space. The results above says that they are asymptotically equivalent. To implement the estimation procedure above, one needs to choose the order $m$ and the number of knots $k_{n}$. The selection of $m$ should 
usually be decided based on the smoothness of the true baseline cumulative hazard functions, and in general, either quadratic or cubic spline functions work sufficiently well in practice. For a given problem, a simple method is to try different values of $m$ and $k_{n}$ and compare the obtained results. To be more objective, an alternative is to apply the Akaike information criterion (AIC) to choose $m$ and $k_{n}$ that give the smallest AIC and more comments on this are given below. We will sketch the proofs of the theorems above in the Appendix.

For inference, once $\hat{\beta}$ and $\hat{\gamma}$ are determined, it is apparent that one needs to estimate their covariance matrix or $\Sigma$. For this, two approaches are commonly used. One is the profile likelihood approach that needs the determination of the profile likelihood of $\beta$ and $\gamma$ and then employ the the inverse of the observed information matrix of the profile likelihood. The other approach is to directly employ the inverse of the observed information matrix of the likelihood function $L(\theta)$ by treating $\Lambda_{1 n}$ and $\Lambda_{2 n}$ as finite-dimensional nuisance parameters. It is easy to see that the former may be much more complicated than the latter. More importantly, the latter is straightforward by using the existing procedures such as the function nlm in $\mathrm{R}$, which is also used for the determination of $\hat{\theta}$ in the numerical studies below. In addition, the simulation results below suggest that the latter seems to work well for practical situations.

For the implementation of the estimation procedure above, in the numerical studies below, we used the $\mathrm{R}$ function $\mathrm{nlm}$ to the perform optimization. The $n l m$ function carries out a minimization of the objective function using a Newton-type algorithm (Dennis and Schnabel, 1996). In the function $\mathrm{nlm}$, the line search methods are used to obtain global convergence and under certain proper conditions, the convergence of algorithm can be established (see Theorem 3.6 and Theorem 3.7 in Nocedal and Wright, 2006). The simulation studies indiate that it seems to work well and reasonably fast.

\section{A Simulation Study}

In this section, we report some results obtained from an extensive simulation study conducted to assess the finite sample properties of the estimation procedure given in the previous sections. In the study, we considered the following three copula models

$$
\begin{gathered}
C_{\alpha}(u, v)=\exp \left\{-\left[(-\log u)^{\alpha}+(-\log v)^{\alpha}\right]^{1 / \alpha}\right\}, \alpha \geq 1, \\
C_{\alpha}(u, v)=u v+\alpha u v(1-u)(1-v),-1 \leq \alpha \leq 1,
\end{gathered}
$$


and

$$
C_{\alpha}(u, v)=\log _{\alpha}\left\{1+\frac{\left(\alpha^{u}-1\right)\left(\alpha^{v}-1\right)}{\alpha-1}\right\}, \alpha \geq 0, \alpha \neq 1 .
$$

In the literature, they are usually referred to as Gumbel, FGM and Frank models, respectively. Note that as mentioned above, here the association parameter $\alpha$ represents the correlation between the failure time of interest $T$ and the censoring interval length $W$. Since the range of $\alpha$ is different for different copula models, we follow the convention of using the Kendall's $\tau$ instead. For the three models above, the relationship between $\alpha$ and $\tau$ is $\tau=1-1 / \alpha, \tau=2 \alpha / 9$, and $\tau=1+4 \rho^{-1}\left\{D_{1}(\rho)-\right.$ $1\}$, respectively. Here $\rho=-\log \alpha$ and $D_{1}(\rho)=\rho^{-1} \int_{0}^{\rho} t\left(e^{t}-1\right)^{-1} d t$.

For the study, we suppose that the covariate $Z_{i}$ 's are binary variables taking value 0 or 1 with the probability 0.5 . For the generation of the observed data, we first generate the failure time $T_{i}$ with $\Lambda_{10}(t)=t$ and then solve the equation

$$
P\left(W \leq w_{i} \mid T_{i}=t_{i}, Z_{i}=z_{i}\right)=\left.\frac{\partial C_{\alpha}(u, v)}{\partial u}\right|_{u=F_{T}\left(t_{i}\right), v=F_{W}\left(w_{i}\right)}=a,
$$

for $w_{i}$ given $T_{i}$ and a random number $a$ generated from the uniform distribution $U(0,1)$. Here we took $\Lambda_{20}(w)=2 w$. Given $W_{i}=w_{i}$, we define the censoring interval by letting $l_{i}$ be the largest number in $\left\{0, w_{i}, 2 w_{i}, 3 w_{i}, \ldots\right\}$ that is smaller than $\min \left\{T_{i}, \zeta_{i}\right\}$. If $l_{i}+w_{i} \leq \zeta_{i}$, then $\delta_{i}=1$ with $r_{i}=l_{i}+w_{i}$ and otherwise, $\delta_{i}=0$. Constant censoring times $\zeta_{i}=\zeta$ were used for all results. The results below are based on 1000 replications with the sample size $n=200$.

Table $\mathbb{U}$ presents the results on estimation of regression parameters when the copula model is correctly specified. The results include the estimated bias (Bias) given by the average of the estimators minus the true value, the sample standard deviation (SSE) of the estimators, the average of the estimated standard errors (SEE), and the $95 \%$ empirical coverage probability (CP). Quadratic splines with $k_{n}=4$ interior knots were used for estimation of both $\Lambda_{10}(t)$ and $\Lambda_{20}(w)$. For $\Lambda_{10}(t)$, the interior knots were taken to be the $0.2,0.4,0.6$ and 0.8 quantiles of the pooled set of all $l_{i}$ 's and the observed $r_{i}$ 's, while for $\Lambda_{20}(w)$, the $0.2,0.4,0.6$ and 0.8 quantiles of the pooled set of the observed $w_{i}$ 's and the $\left(\zeta-l_{i}\right)$ 's from right-censored subjects were used.

The results suggest that the proposed estimation procedure seems to work well for the situations considered here. More specifically, the estimator seems to be unbiased and the variance estimation appears to be reasonable. The results also indicate that the normal approximation to the distribution of the estimated regression parameters appears to be appropriate. The Q-Q plots, which are not shown here, suggest the same. Note that in addition to the hazard function used above, we 
Table 1: Simulation results on estimation of regression parameters with correctly specified copula models and constant baseline hazard functions.

\begin{tabular}{|c|c|c|c|c|c|c|c|c|c|c|c|}
\hline \multicolumn{12}{|c|}{ Under Gumbel copula } \\
\hline & \multicolumn{5}{|c|}{$\tau=0.2$} & \multicolumn{6}{|c|}{$\tau=0.5$} \\
\hline & True & Bias & SSE & SEE & $\mathrm{CP}$ & & True & Bias & SSE & SEE & $\mathrm{CP}$ \\
\hline$\beta$ & 0 & -0.008 & 0.160 & 0.157 & 0.950 & $\beta$ & 0.2 & -0.005 & 0.155 & 0.152 & 0.950 \\
\hline$\gamma$ & 0.2 & -0.005 & 0.160 & 0.158 & 0.933 & $\gamma$ & 0.2 & 0.018 & 0.154 & 0.151 & 0.942 \\
\hline \multicolumn{12}{|c|}{ Under FGM copula } \\
\hline \multicolumn{6}{|c|}{$\tau=-0.1$} & \multicolumn{6}{|c|}{$\tau=0.1$} \\
\hline & True & Bias & SSE & SEE & $\mathrm{CP}$ & & True & Bias & SSE & SEE & $\mathrm{CP}$ \\
\hline$\beta$ & 0 & -0.005 & 0.160 & 0.160 & 0.960 & $\beta$ & 0 & -0.002 & 0.165 & 0.161 & 0.952 \\
\hline$\gamma$ & 0.2 & 0.009 & 0.158 & 0.157 & 0.956 & $\gamma$ & 0.2 & 0.003 & 0.167 & 0.163 & 0.953 \\
\hline \multicolumn{12}{|c|}{ Under Frank copula } \\
\hline & \multicolumn{5}{|c|}{$\tau=-0.5$} & \multicolumn{6}{|c|}{$\tau=0.5$} \\
\hline & True & Bias & SSE & SEE & $\mathrm{CP}$ & & True & Bias & SSE & SEE & $\mathrm{CP}$ \\
\hline$\beta$ & 0.2 & -0.006 & 0.147 & 0.143 & 0.948 & $\beta$ & 0 & -0.015 & 0.168 & 0.156 & 0.937 \\
\hline$\gamma$ & -0.2 & 0.011 & 0.144 & 0.145 & 0.952 & $\gamma$ & 0.2 & -0.015 & 0.179 & 0.162 & 0.933 \\
\hline
\end{tabular}


Table 2: Simulation results on estimation of regression parameters with correctly specified copula models and nonconstant baseline hazard functions.

\begin{tabular}{|c|c|c|c|c|c|c|c|c|c|c|c|}
\hline \multicolumn{12}{|c|}{ Under Gumbel copula } \\
\hline & \multicolumn{5}{|c|}{$\tau=0.2$} & \multicolumn{6}{|c|}{$\tau=0.5$} \\
\hline & True & Bias & SSE & SEE & $\mathrm{CP}$ & & True & Bias & SSE & SEE & $\mathrm{CP}$ \\
\hline$\beta$ & 0 & 0.023 & 0.165 & 0.163 & 0.943 & $\beta$ & 0.2 & -0.000 & 0.165 & 0.161 & 0.947 \\
\hline$\gamma$ & 0.2 & -0.002 & 0.165 & 0.162 & 0.951 & $\gamma$ & 0.2 & 0.051 & 0.142 & 0.160 & 0.978 \\
\hline \multicolumn{12}{|c|}{ Under FGM copula } \\
\hline \multicolumn{6}{|c|}{$\tau=-0.1$} & \multicolumn{6}{|c|}{$\tau=0.1$} \\
\hline & True & Bias & SSE & SEE & $\mathrm{CP}$ & & True & Bias & SSE & SEE & $\mathrm{CP}$ \\
\hline$\beta$ & 0 & -0.005 & 0.169 & 0.167 & 0.949 & $\beta$ & 0 & 0.019 & 0.173 & 0.166 & 0.940 \\
\hline$\gamma$ & 0.2 & 0.005 & 0.162 & 0.163 & 0.948 & $\gamma$ & 0.2 & 0.016 & 0.174 & 0.168 & 0.938 \\
\hline \multicolumn{12}{|c|}{ Under Frank copula } \\
\hline & \multicolumn{5}{|c|}{$\tau=-0.5$} & \multicolumn{6}{|c|}{$\tau=0.5$} \\
\hline & True & Bias & SSE & SEE & $\mathrm{CP}$ & & True & Bias & SSE & SEE & $\mathrm{CP}$ \\
\hline$\beta$ & 0.2 & 0.044 & 0.171 & 0.174 & 0.944 & $\beta$ & 0 & 0.030 & 0.192 & 0.179 & 0.934 \\
\hline$\gamma$ & -0.2 & 0.052 & 0.161 & 0.186 & 0.978 & $\gamma$ & 0.2 & -0.026 & 0.181 & 0.191 & 0.956 \\
\hline
\end{tabular}

also considered the use of other baseline hazard functions for generating failure times. For example,

Table $\square$ presents the estimation results on the regression parameters with $\Lambda_{10}(t)=t^{2} / 4+t / 2$ and $\Lambda_{20}(w)=w^{2} / 2+w$, and they gave similar conclusions to those in Table 1.

Note that in the estimation procedure proposed above, we have assumed that the copula model is known and of course this may not be true in practice. On this, a question of practical interest is possible model misspecification and we also performed some simulation studies to investigate it. Table 3 presents some results obtained under two situations with constant baseline hazard functions. One is that we generated the data from the Gumbel copula with the relatively strong association, but performed the analysis by assuming that they arose from the Frank copula. The other is to generate the data from the FGM copula with weak association, but carry out the analysis by treating them from the Frank copula. In both cases, for comparison, we also considered 
the use of the naive approach that ignores the dependent censoring and included the estimation results in the table. In addition, to evaluate the proposed AIC-based model selection procedure, we calculated the percentages of times when the right model was picked (PRP) among 1000 simulation replications and reported them in Table $\mathbf{3}$. One can see from the upper two panels of Table $\mathbf{3}$ that when the true dependence is relatively strong, the analysis with the misspecified copula model or the naive method seems to yield significant estimation biases of regression parameter $\beta$. For the estimation of regression parameter $\gamma$, the analysis with misspecifed copula model seems to yield strongly significant estimation bias, while the results with naive analysis seems comparable to that using correct copula model. This might be because the interval widths have right-censored data with part of the widths exactly observed, thus independent analysis of it does not lose much information. Please note that our primary interest lies in estimation of $\beta$. Thus we consider that the analysis with correct model outperforms the naive analysis. In this case with relatively strong dependence, the AIC based model selection approach was able to pick up the correct Gumbel copula $97.0 \%$ and $95 \%$ of the times for the two parameter settings, relatively, and picked Frank copula for the rest times. For the situation where the true dependence is weak, as indicated by the results in the lower panel of Table $\mathbf{B}$, the estimators seem to be insensitive to the misspecification of copula models and the naive method seems to perform reasonably well as expected. In this case, the AIC model selection approach picked up the correct FGM copula $48.9 \%$ of the times and the Frank copula $51.1 \%$ times as expected since all copulas are similar when the association is weak. We also considered some other set-ups and obtained similar results.

\section{An Illustrative Example}

To illustrate the estimation procedure developed in the previous sections, we apply it to a set of interval-censored data analyzed by Zhang et al. (2007) among others. One purpose of the study was to investigate the relationship between cytomeglovirus (CMV) shedding and baseline CD4 cell counts and in the study, the patients were followed and tested for CMV shedding in their blood and urine periodically. Since some of the patients missed some of the predetermined visits and returned with their blood and/or urine CMV shedding status changed, only interval-censored data were observed for the times to CMV shedding. In addition to the observed intervals on CMV shedding times, the baseline CD4 cell count was also obtained for each patient. 
Table 3: Simulation results on estimation of regression parameters with mis-specified copula model or naive analysis ignoring dependent censoring and constant baseline hazard functions.

Data generated from Gumbel copula with $\tau=0.5$

\begin{tabular}{rrrrrrrrrrrrrrr} 
Analysis & \multicolumn{3}{c}{ using correct copula } & \multicolumn{4}{c}{ using Frank copula } & \multicolumn{4}{c}{ Naive analysis } \\
& True & Bias & SSE & SEE & CP & Bias & SSE & SEE & CP & Bias & SSE & SEE & CP \\
$\beta$ & 0.2 & -0.005 & 0.155 & 0.152 & 0.950 & 0.014 & 0.171 & 0.160 & 0.937 & -0.026 & 0.162 & 0.162 & 0.954 \\
$\gamma$ & 0.2 & 0.018 & 0.154 & 0.151 & 0.942 & 0.038 & 0.173 & 0.164 & 0.936 & 0.008 & 0.157 & 0.161 & 0.959 \\
& & & & \multicolumn{4}{c}{ PRP $=97 \%$} \\
\hline
\end{tabular}

Data generated from Gumbel copula with $\tau=0.5$

\begin{tabular}{rrrrrrrrrrrrrrr} 
Analysis & \multicolumn{3}{c}{ using correct copula } & \multicolumn{4}{c}{ using Frank copula } & \multicolumn{4}{c}{ Naive analysis } \\
& True & Bias & SSE & SEE & CP & Bias & SSE & SEE & CP & Bias & SSE & SEE & CP \\
$\beta$ & 0.5 & -0.015 & 0.160 & 0.153 & 0.945 & 0.038 & 0.174 & 0.161 & 0.922 & -0.063 & 0.164 & 0.164 & 0.934 \\
$\gamma$ & 0.5 & 0.051 & 0.159 & 0.153 & 0.925 & 0.102 & 0.176 & 0.165 & 0.892 & 0.031 & 0.165 & 0.163 & 0.946 \\
& & & & \multicolumn{4}{c}{ PRP $=95.2 \%$} \\
\hline
\end{tabular}

Data generated from FGM copula with $\tau=0.1$

\begin{tabular}{rrrrrrrrrrrrrr} 
Analysis & \multicolumn{3}{c}{ using correct copula } & \multicolumn{4}{c}{ using Frank copula } & \multicolumn{4}{c}{ Naive analysis } \\
& True & Bias & SSE & SEE & CP & Bias & SSE & SEE & CP & Bias & SSE & SEE & CP \\
$\beta$ & 0 & -0.002 & 0.165 & 0.161 & 0.952 & -0.002 & 0.165 & 0.161 & 0.953 & -0.008 & 0.163 & 0.161 & 0.954 \\
$\gamma$ & 0.2 & 0.003 & 0.167 & 0.163 & 0.953 & 0.003 & 0.166 & 0.163 & 0.954 & 0.000 & 0.162 & 0.161 & 0.957 \\
& & & \multicolumn{4}{c}{ PRP $=48.9 \%$} \\
\hline
\end{tabular}


For the analysis, by following Zhang et aI. (2007), we will dichotomize the baseline CD4 cell the proportional structure. Thus our conclusion of the relatively strong association between CMV shedding in urine and visit compliance seems more convincing. More discussions can be found in Section 6. 

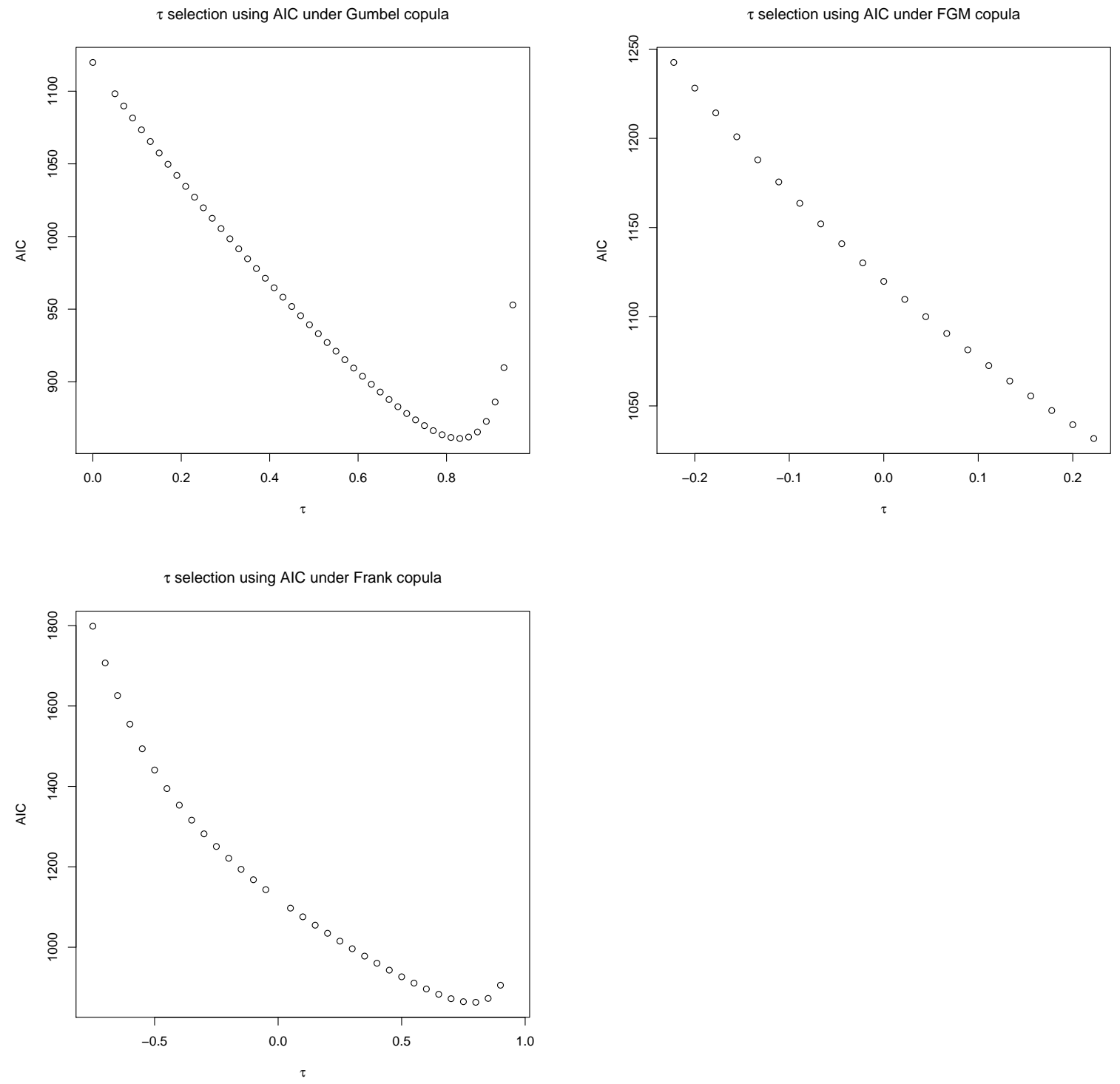

Figure 2: AIC values at a grid of $\tau$ values under Gumbel, FGM and Frank copulas. 
Table 4: Estimated treatment effects for CMV shedding in urine.

\begin{tabular}{ccccccccc}
\hline copula & $\tau$ & $\hat{\beta}$ & SEE & $p$-value & $\hat{\gamma}$ & SEE & $p$-value & AIC \\
\hline Gumbel & 0 & 0.89 & 0.20 & $<0.0001$ & 0.57 & 0.19 & 0.0033 & 1119.7980 \\
& 0.25 & 0.96 & 0.20 & $<0.0001$ & 0.67 & 0.20 & 0.0006 & 1019.7581 \\
& 0.5 & 0.89 & 0.19 & $<0.0001$ & 0.65 & 0.19 & 0.0006 & 936.1881 \\
& 0.75 & 0.73 & 0.18 & $<0.0001$ & 0.57 & 0.18 & 0.0019 & 869.8624 \\
& 0.83 & 0.64 & 0.18 & 0.0004 & 0.52 & 0.18 & 0.0043 & 861.0044 \\
FGM & $-2 / 9$ & 0.79 & 0.23 & 0.0004 & 0.43 & 0.21 & 0.0421 & 1242.5600 \\
& $-1 / 9$ & 0.86 & 0.20 & $<0.0001$ & 0.54 & 0.20 & 0.0071 & 1175.5460 \\
& 0 & 0.89 & 0.20 & $<0.0001$ & 0.57 & 0.19 & 0.0033 & 1119.7980 \\
& $1 / 9$ & 0.89 & 0.20 & $<0.0001$ & 0.57 & 0.19 & 0.0028 & 1072.5910 \\
& $2 / 9$ & 0.87 & 0.20 & $<0.0001$ & 0.56 & 0.19 & 0.0031 & 1031.7420 \\
Frank & -0.5 & 0.61 & 0.24 & 0.0115 & 0.35 & 0.23 & 0.1363 & 1440.8235 \\
& -0.25 & 0.80 & 0.21 & 0.00001 & 0.51 & 0.20 & 0.0127 & 1250.6426 \\
& 0 & 0.89 & 0.20 & $<0.0001$ & 0.57 & 0.19 & 0.0033 & 1119.7980 \\
& 0.25 & 0.87 & 0.19 & $<0.0001$ & 0.58 & 0.19 & 0.0019 & 1015.2402 \\
& 0.5 & 0.80 & 0.18 & $<0.0001$ & 0.56 & 0.18 & 0.0016 & 926.5547 \\
& 0.75 & 0.69 & 0.18 & 0.0001 & 0.54 & 0.18 & 0.0020 & 864.6551 \\
& 0.79 & 0.66 & 0.18 & 0.0003 & 0.54 & 0.18 & 0.0027 & 862.8108 \\
\hline
\end{tabular}


Table 1 gives the analysis results on the CMV shedding time in blood with $k_{n}=3$. As above, we also performed the analysis by using different numbers of knots under each of the three copula models and the smallest AIC value is given by the estimation under the Gumbel copula model with $k_{n}=3$ and $\tau=0.93$. For the case, we obtained $\hat{\beta}=1.11$ with the estimated standard error being 0.42 , corresponding to a $p$-value of 0.0079 for testing $\beta=0$. In comparison, Zhang et al. (2007)

gave $\hat{\beta}=0.81$ with the standard error of 0.33 and the $p$-value of 0.014 , and both methods suggest some significant effect of the baseline CD4 count on the CMV shedding time. Also the proposed approach indicates that the censoring mechanism seems to be positively related with the CMV shedding in blood.

Note that in the proposed estimation approach, the marginal proportional hazards model is used for both the failure time of interest $T$ and the observation interval length $W$. To complete the analysis, one may also want to verify these assumptions. For $W$, since right-censored data are observed, the model check is straightforward and actually we obtained the $p$-values of 0.064 and 0.495 for CMV shedding in blood and urine, respectively. They suggest that the proportional hazards model seems to be appropriate.

\section{Discussion and Concluding Remarks}

In the preceding sections, an approach has been proposed for regression analysis of intervalcensored failure time data when the failure time of interest and the censoring mechanism may be dependent through the length of censoring intervals. As mentioned before, such data occur quite often in medical studies and other investigations although only limited research exists due to the difficulties involved. Also as mentioned above, for the analysis, we have assumed that the copula model and the association parameter are known as they are not identifiable in general without extra information or data. In order to perform the analysis, one way is to try different models and parameter values similarly to a sensitivity analysis. Also as done in Sections 4 and 5, one could apply the AIC for the determination or selection of appropriate copula model and association parameter. The same issue occurs in many other areas too such as in tumorigenecity experiments (Lagakos and Louis, 1988).

In the proposed method, we have employed the copula model approach and $I$-spline functions. With respect to the former, as pointed above, another commonly used approach for modeling correlated variables is through the use of latent variables, which often requires some complicated 
Table 5: Estimated treatment effects for CMV shedding in blood.

\begin{tabular}{ccccccccc}
\hline copula & $\tau$ & $\hat{\beta}$ & SEE & $p$-value & $\hat{\gamma}$ & SEE & $p$-value & AIC \\
\hline Gumbel & 0 & 1.20 & 0.42 & 0.0039 & 0.77 & 0.41 & 0.0637 & 544.6019 \\
& 0.25 & 1.25 & 0.43 & 0.0040 & 0.82 & 0.43 & 0.0555 & 500.5852 \\
& 0.5 & 1.25 & 0.44 & 0.0049 & 0.88 & 0.43 & 0.0428 & 457.0369 \\
& 0.75 & 1.17 & 0.43 & 0.0063 & 0.92 & 0.42 & 0.0295 & 412.4883 \\
& 0.93 & 1.11 & 0.42 & 0.0079 & 1.00 & 0.42 & 0.0160 & 387.0831 \\
FGM & $-2 / 9$ & 1.11 & 0.41 & 0.0062 & 0.67 & 0.39 & 0.0841 & 625.1859 \\
& $-1 / 9$ & 1.19 & 0.41 & 0.0041 & 0.74 & 0.41 & 0.0734 & 574.0249 \\
& 0 & 1.20 & 0.42 & 0.0039 & 0.77 & 0.41 & 0.0637 & 544.6019 \\
& $1 / 9$ & 1.22 & 0.42 & 0.0035 & 0.79 & 0.41 & 0.0567 & 523.3384 \\
& $2 / 9$ & 1.23 & 0.42 & 0.0031 & 0.81 & 0.41 & 0.0499 & 506.2520 \\
Frank & -0.5 & 1.17 & 0.41 & 0.0041 & 0.71 & 0.41 & 0.0822 & 726.6607 \\
& -0.25 & 1.19 & 0.41 & 0.0042 & 0.73 & 0.41 & 0.0773 & 611.5466 \\
& 0 & 1.20 & 0.42 & 0.0039 & 0.77 & 0.41 & 0.0637 & 544.6019 \\
& 0.25 & 1.23 & 0.42 & 0.0031 & 0.82 & 0.41 & 0.0455 & 496.9193 \\
& 0.5 & 1.24 & 0.41 & 0.0025 & 0.89 & 0.41 & 0.0283 & 457.4012 \\
& 0.75 & 1.17 & 0.39 & 0.0030 & 0.91 & 0.39 & 0.0178 & 418.3175 \\
& 0.94 & 1.04 & 0.36 & 0.0041 & 0.92 & 0.35 & 0.0085 & 388.7243 \\
\hline
\end{tabular}



11371062) to the second author and a NSF grant to the third author.

\section{Appendix A. Proofs of Theorems $1-3$}

For the proof of Theorems $1-3$, we need the following regularity conditions.

(A1) The covariate $Z$ has a bounded support in $R^{p}$, where $p$ is the dimension of $Z$. and $\partial C_{\alpha}(u, v) / \partial v$ are Lipschitz. 
(A3) Let $l(\theta, X)$ denote the log likelihood function based on the single observation $X=(\delta, L, R, Z, \zeta)$ as defined in Section 2. For any positive number $\epsilon$, we have that

$$
\sup _{d\left(\theta, \theta_{0}\right)>\epsilon} P l(\theta, X)<P l\left(\theta_{0}, X\right) .
$$

(A4) For $k=1,2$, the $m$ th derivative of $\Lambda_{k}(\cdot)$, denoted by $\Lambda_{k}^{(m)}(\cdot)$, is Holder continuous with exponent $\eta$. That is, there exist a positive real value $M$ and some $\eta \in(0,1]$ such that $\mid \Lambda_{k}^{(m)}\left(t_{1}\right)-$ $\Lambda_{k}^{(m)}\left(t_{2}\right)|\leq M| t_{1}-\left.t_{2}\right|^{\eta}$ for all $t_{1}, t_{2} \in(l, u), k=1,2$. In the following, we denote $r=m+\eta$. (A5) The matrix $E\left(S_{\vartheta} S_{\vartheta}^{\prime}\right)$ is finite and positive definite with $\vartheta=\left(\beta^{\prime}, \gamma^{\prime}\right)^{\prime}$, where $S_{\vartheta}$ is defined in the proof of Theorem 3 below.

These conditions are mild and usually satisfied in practice (Huang and Rossini, 11997; Zhang et al, 2010$)$. In the following proofs of Theorems $1-3$, the empirical process theory and some techniques commonly used in nonparametric literature will be involved. For ease of exposition, we denote $P g=\int g(x) d P(x)$ and $P_{n} g=n^{-1} \sum_{i=1}^{n} g\left(X_{i}\right)$, the empirical process indexed by function $g(X)$. And we let $K, M, M_{n}, C$ be generic constants in the following and could vary from place to place.

Proof of Theorem 1: To establish the consistency, we will employ the empirical process theory and need the following two lemmas, whose proofs are given in the Supporting Information to save the space.

Lemma 1 (Calculation of the covering number ). Under Conditions (A1) and (A2), the covering number of the class $\mathcal{L}_{n}=\left\{l(\theta, X): \theta \in \Theta_{n}\right\}$ satisfies

$$
N\left(\epsilon, \mathcal{L}_{n}, L_{1}\left(P_{n}\right)\right) \leq K M^{2 p} M_{n}^{2\left(m+k_{n}\right)} \epsilon^{-p_{m}},
$$

where $p_{m}=2 p+2\left(m+k_{n}\right)$.

Lemma 2 (Uniform convergence). Under Conditions (A1) and (A2), we have

$$
\sup _{\theta \in \Theta_{n}}\left|P_{n} l(\theta, X)-P l(\theta, X)\right| \rightarrow 0,
$$

almost surely.

For the proof, consider the class $\mathcal{L}_{n}$ and note that according to Lemma 1, the covering number of the class $\mathcal{L}_{n}$ satisfies

$$
N\left(\epsilon, \mathcal{L}_{n}, L_{1}\left(P_{n}\right)\right) \leq K M^{2 p} M_{n}^{2\left(m+k_{n}\right)} \epsilon^{-p_{m}},
$$


where $p_{m}=2 p+2\left(m+k_{n}\right)$. Furthermore, by Lemma 2 , we have

$$
\sup _{\theta \in \Theta_{n}}\left|P_{n} l(\theta, X)-P l(\theta, X)\right| \rightarrow 0
$$

almost surely. Let $M(\theta, X)=-l(\theta, X), \zeta_{1 n}=\sup _{\theta \in \Theta_{n}}\left|P_{n} M(\theta, X)-P M(\theta, X)\right|, \zeta_{2 n}=P_{n} M\left(\theta_{0}, X\right)-$ $P M\left(\theta_{0}, X\right)$, and denote $K_{\epsilon}=\left\{\theta: d\left(\theta, \theta_{0}\right) \geq \epsilon, \theta \in \Theta_{n}\right\}$. By (A.]), we have

$$
\begin{aligned}
\inf _{K_{\epsilon}} P M(\theta, X) & =\inf _{K_{\epsilon}}\left\{P M(\theta, X)-P_{n} M(\theta, X)+P_{n} M(\theta, X)\right\} \\
& \leq \zeta_{1 n}+\inf _{K_{\epsilon}} P_{n} M(\theta, X) .
\end{aligned}
$$

If $\hat{\theta}_{n} \in K_{\epsilon}$, we can get that

$$
\inf _{K_{\epsilon}} P_{n} M(\theta, X)=P_{n} M\left(\hat{\theta}_{n}, X\right) \leq P_{n} M\left(\theta_{0}, X\right)=\zeta_{2 n}+P M\left(\theta_{0}, X\right) .
$$

With condition (A3), we obtain that $\inf _{K_{\epsilon}} P M(\theta, X)-P M\left(\theta_{0}, X\right)=\delta_{\epsilon}>0$. Further analysis by using (A.2) and (A.3) leads to $\inf _{K_{\epsilon}} P M(\theta, X) \leq \zeta_{1 n}+\zeta_{2 n}+P M\left(\theta_{0}, X\right)=\zeta_{n}+P M\left(\theta_{0}, X\right)$ with $\zeta_{n}=\zeta_{1 n}+\zeta_{2 n}$. Hence, we have $\zeta_{n} \geq \delta_{\epsilon}$ which implies that $\left\{\hat{\theta}_{n} \in K_{\epsilon}\right\} \subseteq\left\{\zeta_{n} \geq \delta_{\epsilon}\right\}$. By (A.]) and strong law of large numbers, we have $\zeta_{1 n}=o(1)$ almost surely and $\zeta_{2 n}=o(1)$ almost surely. Therefore, by $\cup_{k=1}^{\infty} \cap_{n=k}^{\infty}\left\{\hat{\theta}_{n} \in K_{\epsilon}\right\} \subseteq \cup_{k=1}^{\infty} \cap_{n=k}^{\infty}\left\{\zeta_{n} \geq \delta_{\epsilon}\right\}$, we complete the proof.

Proof of Theorem 2

To derive the convergence rate, for any $\eta>0$, define the class $\mathcal{F}_{\eta}=\left\{l\left(\theta_{n 0}, X\right)-l(\theta, X): \theta \in\right.$ $\left.\Theta_{n}, d\left(\theta, \theta_{n 0}\right) \leq \eta\right\}$ with $\theta_{n 0}=\left(\beta_{0}, \gamma_{0}, \Lambda_{1 n 0}, \Lambda_{2 n 0}\right)$. Following the calculation of Shen and Wong (11994, p.597), we can establish that $\log N_{[]}\left(\varepsilon, \mathcal{F}_{\eta},\|\cdot\|_{2}\right) \leq C N \log (\eta / \varepsilon)$ with $N=2\left(m+k_{n}\right)$. Moreover, some algebraic calculations lead to $\left\|l\left(\theta_{n 0}, X\right)-l(\theta, X)\right\|_{2}^{2} \leq C \eta^{2}$ for any $l\left(\theta_{n 0}, X\right)-$ $l(\theta, X) \in \mathcal{F}_{\eta}$.

Therefore, by Lemma 3.4.2 of van der Vaart and Wellner (1996), we can obtain

$$
E_{P}\left\|n^{1 / 2}\left(P_{n}-P\right)\right\|_{\mathcal{F}_{\eta}} \leq C J_{\eta}\left(\varepsilon, \mathcal{F}_{\eta},\|\cdot\|_{2}\right)\left\{1+\frac{J_{\eta}\left(\varepsilon, \mathcal{F}_{\eta},\|\cdot\|_{2}\right)}{\eta^{2} n^{1 / 2}}\right\}
$$

where $J_{\eta}\left(\varepsilon, \mathcal{F}_{\eta},\|\cdot\|_{2}\right)=\int_{0}^{\eta}\left\{1+\log N_{[]}\left(\varepsilon, \mathcal{F}_{\eta},\|\cdot\|_{2}\right)\right\}^{1 / 2} d \varepsilon \leq C N^{1 / 2} \eta$. The right-hand side of $r_{n}^{2} \phi_{n}\left(1 / r_{n}\right)=r_{n} N^{1 / 2}+r_{n}^{2} N / n^{1 / 2}<2 n^{1 / 2}$, where $r_{n}=N^{-1 / 2} n^{1 / 2}=n^{(1-\nu) / 2}$ with $0<\nu<0.5$. Hence $n^{(1-\nu) / 2} d\left(\hat{\theta}, \theta_{n 0}\right)=O_{P}(1)$ by Theorem 3.2.5 of van der Vaart and Wellner ([1996). This, together with $d\left(\theta_{n 0}, \theta_{0}\right)=O_{p}\left(n^{-r \nu}\right)$ (Lemma A1 in Luet al. (2007)), yields that $d\left(\hat{\theta}, \theta_{0}\right)=$ 
$O_{p}\left(n^{-(1-\nu) / 2}+n^{-r \nu}\right)$. The choice of $\nu=1 /(1+2 r)$ yields the rate of convergence of $d\left(\hat{\theta}_{n}, \theta_{0}\right)=$ $O_{p}\left(n^{-\frac{r}{1+2 r}}\right)$.

\section{Proof of Theorem 3}

Denote $V$ as the linear span of $\Theta_{0}-\theta_{0}$, where $\theta_{0}$ denotes the true value of $\theta=(\beta, \gamma, \varphi)$ and $\Theta_{0}$ the true parameter space. Let $l(\theta, X)$ be the log-likelihood for a sample of size one and $\delta_{n}=\left(n^{-(1-\nu) / 2}+n^{-r \nu}\right)$. For any $\theta \in\left\{\theta \in \Theta_{0}: d\left(\theta, \theta_{0}\right)=O\left(\delta_{n}\right)\right\}$, define the first order directional derivative of $l(\theta, X)$ at the direction $v \in V$ as

$$
\dot{l}(\theta, X)[v]=\left.\frac{d l(\theta+s v, X)}{d s}\right|_{s=0},
$$

and the second order directional derivative as

$$
\ddot{l}(\theta, X)[v, \tilde{v}]=\left.\left.\frac{d^{2} l(\theta+s v+\tilde{s} \tilde{v}, X)}{d \tilde{s} d s}\right|_{s=0}\right|_{\tilde{s}=0}=\left.\frac{d \dot{l}(\theta+\tilde{s} \tilde{v}, X)}{d \tilde{s}}\right|_{\tilde{s}=0} .
$$

Define the Fisher inner product on the space $V$ as $<v, \tilde{v}>=P\{i(\theta, X)[v] i(\theta, X)[\tilde{v}]\}$ and the Fisher norm for $v \in V$ as $\|v\|^{1 / 2}=\langle v, v>$. Let $\bar{V}$ be the closed linear span of $V$ under the Fisher norm. Then $(\bar{V},\|\cdot\|)$ is a Hilbert space. Also define the smooth functional of $\theta$ as $\gamma(\theta)=b_{1}^{\prime} \beta+b_{2}^{\prime} \gamma$, where $b=\left(b_{1}^{\prime}, b_{2}^{\prime}\right)^{\prime}$ is any vector of $2 p$ dimension with $\|b\| \leq 1$. For any $v \in V$, we denote

$$
\dot{\gamma}\left(\theta_{0}\right)[v]=\left.\frac{d \gamma\left(\theta_{0}+s v\right)}{d s}\right|_{s=0}=r(v),
$$

whenever the right hand-side limit is well defined. Note that $\gamma(\theta)-\gamma\left(\theta_{0}\right)=\dot{\gamma}\left(\theta_{0}\right)\left[\theta-\theta_{0}\right]$. It follows by the Riesz representation theorem that there exists $v^{*} \in \bar{V}$ such that $\dot{\gamma}\left(\theta_{0}\right)[v]=\left\langle v^{*}, v\right\rangle$ for all $v \in \bar{V}$ and $\left\|v^{*}\right\|^{2}=\left\|\dot{\gamma}\left(\theta_{0}\right)\right\|$.

Let $\varepsilon_{n}$ be any positive sequence satisfying $\varepsilon_{n}=o\left(n^{-1 / 2}\right)$. For any $v^{*} \in \Theta_{0}$, by (A4) and Corollary 6.21 of Schumaker (11981, p.277), there exists $\Pi_{n} v^{*} \in \Theta_{n}$ such that $\left\|\Pi_{n} v^{*}-v^{*}\right\|=o(1)$ and $\delta_{n}\left\|\Pi_{n} v^{*}-v^{*}\right\|=o\left(n^{-1 / 2}\right)$. Also define $r\left[\theta-\theta_{0}, X\right]=l(\theta, X)-l\left(\theta_{0}, X\right)-\dot{l}(\theta, X)\left[\theta-\theta_{0}\right]$. Then 
by definition of $\hat{\theta}$, we have

$$
\begin{aligned}
0 & \quad P_{n}\left[l(\hat{\theta}, X)-l\left(\hat{\theta} \pm \varepsilon_{n} \Pi_{n} v^{*}, X\right)\right] \\
= & \left(P_{n}-P\right)\left[l(\hat{\theta}, X)-l\left(\hat{\theta} \pm \varepsilon_{n} \Pi_{n} v^{*}, X\right)\right]+P\left[l(\hat{\theta}, X)-l\left(\hat{\theta} \pm \varepsilon_{n} \Pi_{n} v^{*}, X\right)\right] \\
= & \pm \varepsilon_{n} P_{n} \dot{l}(\theta, X)\left[\Pi_{n} v^{*}\right]+\left(P_{n}-P\right)\left\{r\left[\theta-\theta_{0}, X\right]-r\left[\hat{\theta} \pm \varepsilon_{n} \Pi_{n} v^{*}-\theta_{0}, X\right]\right\} \\
& \quad+P\left\{r\left[\theta-\theta_{0}, X\right]-r\left[\hat{\theta} \pm \varepsilon_{n} \Pi_{n} v^{*}-\theta_{0}, X\right]\right\} \\
= & \mp \varepsilon_{n} P_{n} \dot{l}(\theta, X)\left[v^{*}\right] \pm \varepsilon_{n} P_{n} \dot{l}(\theta, X)\left[\Pi_{n} v^{*}-v^{*}\right]+\left(P_{n}-P\right)\left\{r\left[\theta-\theta_{0}, X\right]\right. \\
& \left.\quad-r\left[\hat{\theta} \pm \varepsilon_{n} \Pi_{n} v^{*}-\theta_{0}, X\right]\right\}+P\left\{r\left[\theta-\theta_{0}, X\right]-r\left[\hat{\theta} \pm \varepsilon_{n} \Pi_{n} v^{*}-\theta_{0}, X\right]\right\} \\
& \quad=\quad \mp \varepsilon_{n} P_{n} \dot{l}(\theta, X)\left[v^{*}\right]+I_{1}+I_{2}+I_{3} .
\end{aligned}
$$

For $I_{1}$, with conditions (A1)-(A2), Chebyshev inequality and $\left\|\Pi_{n} v^{*}-v^{*}\right\|=o(1)$, we can conclude that $I_{1}=o_{p}\left(n^{-1 / 2}\right) \varepsilon_{n}$. For $I_{2}$, we have

$$
\begin{aligned}
I_{2} & =\left(P_{n}-P\right)\left\{l(\hat{\theta}, X)-l\left(\hat{\theta} \pm \varepsilon_{n} \Pi_{n} v^{*}, X\right) \pm \varepsilon_{n} l\left(\theta_{0}, X\right)\left[\Pi_{n} v^{*}\right]\right\} \\
& =\mp \varepsilon_{n}\left(P_{n}-P\right)\left\{i(\tilde{\theta}, X)\left[\Pi_{n} v^{*}\right]-i\left(\theta_{0}, X\right)\left[\Pi_{n} v^{*}\right]\right\},
\end{aligned}
$$
van der Vaart and Wellner ([1996), we have $I_{2}=\varepsilon_{n} \times o_{p}\left(n^{-1 / 2}\right)$.

For $I_{3}$, note that

$$
\begin{aligned}
P\left(r\left[\theta-\theta_{0}, X\right]\right)= & \left.P\left\{l(\theta, X)-l\left(\theta_{0}, X\right)-\dot{l}\left(\theta_{0}, X\right)\left[\theta-\theta_{0}\right]\right)\right\} \\
= & 2^{-1} P\left\{\ddot{l}(\tilde{\theta}, X)\left[\theta-\theta_{0}, \theta-\theta_{0}\right]-\ddot{l}\left(\theta_{0}, X\right)\left[\theta-\theta_{0}, \theta-\theta_{0}\right]\right\} \\
& \quad+2^{-1} P\left\{\ddot{l}\left(\theta_{0}, X\right)\left[\theta-\theta_{0}, \theta-\theta_{0}\right]\right\} \\
= & 2^{-1} P\left\{\ddot{l}\left(\theta_{0}, X\right)\left[\theta-\theta_{0}, \theta-\theta_{0}\right]\right\}+\varepsilon_{n} \times o_{p}\left(n^{-1 / 2}\right),
\end{aligned}
$$

where $\tilde{\theta}$ lies between $\theta_{0}$ and $\theta$ and the last equation is due to Taylor expansion, conditions (A1)-(A2) and $r>2$. Therefore,

$$
\begin{aligned}
I_{3} & =-2^{-1}\left\{\left\|\hat{\theta}-\theta_{0}\right\|^{2}-\left\|\hat{\theta} \pm \varepsilon_{n} \Pi_{n} v^{*}-\theta_{0}\right\|^{2}\right\}+\varepsilon_{n} \times o_{p}\left(n^{-1 / 2}\right) \\
& = \pm \varepsilon_{n}<\hat{\theta}-\theta_{0}, \Pi_{n} v^{*}>+2^{-1}\left\|\varepsilon_{n} \Pi_{n} v^{*}\right\|^{2}+\varepsilon_{n} \times o_{p}\left(n^{-1 / 2}\right) \\
& = \pm \varepsilon_{n}<\hat{\theta}-\theta_{0}, v^{*}>+2^{-1}\left\|\varepsilon_{n} \Pi_{n} v^{*}\right\|^{2}+\varepsilon_{n} \times o_{p}\left(n^{-1 / 2}\right) \\
& = \pm \varepsilon_{n}<\hat{\theta}-\theta_{0}, v^{*}>+\varepsilon_{n} \times o_{p}\left(n^{-1 / 2}\right),
\end{aligned}
$$


where the last equality holds because of Cauchy-Schwartz inequality and the facts that $\delta_{n} \| \Pi_{n} v^{*}-$ $v^{*} \|=o\left(n^{-1 / 2}\right)$ and $\left\|\Pi_{n} v^{*}\right\|^{2} \rightarrow\left\|v^{*}\right\|^{2}$. Combing the above facts, together with $\operatorname{Pi}\left(\theta_{0}, X\right)\left[v^{*}\right]=0$, we can establish that

$$
\begin{aligned}
0 & \leq P_{n}\left\{l(\hat{\theta}, X)-l\left(\hat{\theta} \pm \varepsilon_{n} \Pi_{n} v^{*}, X\right)\right\} \\
& =\mp \varepsilon_{n} P_{n} \dot{l}\left(\theta_{0}, X\right)\left[v^{*}\right] \pm \varepsilon_{n}<\hat{\theta}-\theta_{0}, v^{*}>+\varepsilon_{n} \times o_{p}\left(n^{-1 / 2}\right) \\
& =\mp \varepsilon_{n}\left(P_{n}-P\right)\left\{i\left(\theta_{0}, X\right)\left[v^{*}\right]\right\} \pm \varepsilon_{n}<\hat{\theta}-\theta_{0}, v^{*}>+\varepsilon_{n} \times o_{p}\left(n^{-1 / 2}\right) .
\end{aligned}
$$

Therefore, we obtain that $\sqrt{n}<\hat{\theta}-\theta_{0}, v^{*}>=\sqrt{n}\left(P_{n}-P\right)\left\{i\left(\theta_{0}, X\right)\left[v^{*}\right]\right\}+o_{p}(1) \rightarrow N\left(0,\left\|v^{*}\right\|^{2}\right)$, where the asymptotic normality is guaranteed by the central limits theorem and the asymptotic variance being equal to $\left\|v^{*}\right\|^{2}=\left\|\dot{l}\left(\theta_{0}, X\right)[v *]\right\|^{2}$. This together with condition (A5) implies that $n^{1 / 2}\left(\gamma(\hat{\theta})-\gamma\left(\theta_{0}\right)\right)=n^{1 / 2}<\hat{\theta}-\theta_{0}, v^{*}>+o_{p}(1) \rightarrow N\left(0,\left\|v^{*}\right\|^{2}\right)$ in distribution. The semiparametric efficiency can be established by applying the result of Bickel and Kwon (20001) or Theorem 4 in Shen (10997).

For each component $\vartheta_{q}, q=1,2, \cdots, 2 p$, we denote by $\varphi_{q}^{*}=\left(b_{1 q}^{*}, b_{2 q}^{*}\right)$ the solution to

$$
\inf _{\varphi_{q}^{*}} E\left\{l_{\vartheta} \cdot e_{q}-l_{b_{1}^{*}} \cdot b_{1 q}^{*}-l_{b_{2}^{*}} \cdot b_{2 q}^{*}\right\}^{2}
$$

where $l_{\vartheta}=\left(l_{\beta}^{\prime}, l_{\gamma}^{\prime}\right)^{\prime}, l_{b_{1}^{*}}$ and $l_{b_{2}^{*}}$ are the partial derivatives of $l(\theta, X)$ with respect to $b_{1}^{*}$ and $b_{2}^{*}$, respectively. Now let $\varphi^{*}=\left(\varphi_{1}^{*}, \cdots, \varphi_{q}^{*}\right)$. By the calculations of Chen et al. (2006), we have

$$
\left\|v^{*}\right\|^{2}=\left\|\dot{\gamma}\left(\theta_{0}\right)\right\|=\sup _{v \in \bar{V}:\|v\|>0} \frac{\left|\dot{\gamma}\left(\theta_{0}\right)[v]\right|}{\|v\|}=b^{\prime} \Sigma b,
$$

where $\Sigma=E\left(S_{\vartheta} S_{\vartheta}^{\prime}\right), S_{\vartheta}=\left\{l_{\vartheta}-l_{b_{1}^{*}} b_{1}^{*}-l_{b_{2}^{*}} b_{2}^{*}\right\}$. Since $b^{\prime}\left(\left(\hat{\beta}-\beta_{0}\right)^{\prime},\left(\hat{\gamma}-\gamma_{0}\right)^{\prime}\right)=<\hat{\theta}-\theta_{0}, v^{*}>$, the conclusion of the theorem follows by the Cramér-Wold device.

\section{References}

Betensky, R. A. and Finkelstein, D. M. (2002). Testing for dependence between failure time and visit compliance with interval-censored data. Biometrics 58, 58-63.

Bickel, P. J. and Kwon, J. (2001). Inference for semiparametric models: some questions and an answer. Statist. Sinica 11, 863-960.

Chen, X., Fan, Y., and Tsyrennikov, V. (2006). Efficient estimation of semiparametric multivariate copula models. J. Amer. Statist. Assoc. 101, 1228-40. 
Chen, D.-G., Sun, J. and Peace, K. E. (2012). Interval-Censored Time-to-Event Data: Methods and Applications. Chapman \& Hall/CRC.

Chen, Y.-H. (2010). Semiparametric marginal regression analysis for dependent competing risks under an assumed copula. J. R. Stat. Soc. Ser. B Stat. Methodol. 72, 235-51.

Dennis Jr, J. E., and Schnabel, R. B. (1996). Numerical methods for unconstrained optimization and nonlinear equations (Vol. 16). Siam.

Finkelstein, D. M. (1986). A proportional hazards model for interval-censored failure time data. Biometrics 42, 845-54.

Finkelstein, D. M., Goggins, W. B. and Schoenfeld, D. A. (2002). Analysis of failure time data with dependent interval censoring. Biometrics 58, 298-304.

Goethals, K., Janssen, P. and Duchateau, L. (2008). Frailty models and copulas: similarities and differences. Journal of Applied Statistics 35(9), 1071-9.

Goggins, W. B., Finkelstein, D. M., Schoenfeld, D. A. and Zaslavsky, A. M. (1998). A Markov chain Monte Carlo EM algorithm for analyzing interval censored data under the Cox proportional hazards model. Biometrics 54, 1498-507.

Hougaard, P. (2000). Analysis of Multivariate Survival Data. Springer, New York.

Huang, J. (1996). Efficient estimation for the proportional hazards model with interval censoring. Ann. Statist. 24, 540-68

Huang, J. and Rossini, A. J. (1997). Sieve estimation for the proportional odds failure- time regression model with interval censoring. J. Amer. Statist. Assoc. 92, 960-67.

Kalbfleisch, J. D., and Prentice, R. L. (2002). The Statistical Analysis of Failure Time Data, 2nd edn. Wiley, New York.

Lagakos, S. W. and Louis, T. A. (1988). Use of tumor lethality to interpret tumorigenicity experiments lacking cause-of-death data. J. Appl. Stat. 37, 169-79.

Lu, M., Zhang, Y., and Huang, J. (2007). Estimation of the mean function with panel count data using monotone polynomial splines. Biometrika 94(3), 705-18. 
Nelsen, R. B. (2006). An Introduction to Copulas 2nd edn. Springer, New York.

Nocedal, J., and Wright, S. (2006). Numerical Optimization. Springer Science \& Business Media.

Pollard, D. (1984). Convergence of Stochastic Processes. Springer, New York.

Ramsay, J. O. (1988). Monotone regression splines in action. Statist. Sci. 3, 425-41.

Schick, Q. and Yu, Q. (2000) Consistency of the GMLE with Mixed Case Interval-censored Data. Scand. J. Statist. 27, 45-55.

Schumaker, L. L. (1981). Spline Functions: Basic Theory 3rd edn. Cambridge Mathematical Library.

Shen, X. and Wong, W. H. (1994). Convergence rate of sieve estimates. Ann. Statist., 22, 580-615.

Shen, X. (1997). On methods of sieves and penalization. Ann. Statist., 25, 2555-91.

Sun, J. (2006). The Statistical Analysis of Interval-Censored Failure Time Data. Springer, New York.

van de Geer, S. (2000). Empirical Process in M-Estimation. Cambridge University Press.

van der Vaart, A. W. and Wellner, J. A. (1996). Weak Convergence and Empirical Processes. Springer, Berlin.

Zhang, Y., Hua, L., and Huang, J. (2010). A spline-based semiparametric maximum likelihood estimation method for the Cox model with interval-censored data. Scand. J. Stat., 37, 338-54.

Zhang, Z., Sun, L., Zhao X., and Sun, J. (2005). Regression analysis of interval-censored failure time data with linear transformation models. Canad. J. Statist., 33, 61-70.

Zhang, Z., Sun, L., Sun, J. and Finkelstein, D. M. (2007). Regression analysis of failure time data with informative interval censoring. Stat. Med. 26, 2533-46.

Zheng, M., Klein, J. P. (1995). Estimates of marginal survival for dependent competing risk based on an assumed copula. Biometrika, 82, 127-138. 